\title{
Structured doubling algorithms for solving g-palindromic quadratic eigenvalue problems*
}

\author{
Eric King-wah $\mathrm{Chu}^{\dagger} \quad$ Tsung-Ming Huang ${ }^{\ddagger} \quad$ Wen-Wei Lin ${ }^{\S}$
}

\begin{abstract}
The T-palindromic quadratic eigenvalue problem $\left(\lambda^{2} B+\lambda C+A\right) x=0$, with $A, B, C \in \mathbb{C}^{n \times n}$, $C^{T}=C$ and $B^{T}=A$, governs the vibration behavior of trains. One way to solve the problem is to apply a structure-preserving doubling algorithm (SDA) to the nonlinear matrix equation (NME) $X+B X^{-1} A=C$ and "square-root" the matrix quadratic involved. In this paper, we generalize the SDA for the solution of (odd and even) T- and H-palindromic quadratic eigenvalue problems in a unified fashion. A convergence proof and several numerical examples are provided.
\end{abstract}

\section{Introduction}

We consider a quadratic eigenvalue problem (QEP)

$$
Q(\lambda) x \equiv\left(\lambda^{2} B+\lambda C+A\right) x=0,
$$

where $A, B, C \in \mathbb{C}^{n \times n}, \lambda \in \mathbb{C}, x \neq 0 \in \mathbb{C}^{n}$. Many QEPs from a variety of applications have extra structure that results in certain symmetry in the spectrum. One such QEP is the palindromic QEP with the property that reversing the order of the coefficients leads back to the original QEP, which explains the adjective "palindromic".

If $B^{T}=A$ and $C^{T}=C$, then (1) is called a T-palindromic QEP. The T-palindromic QEP was first raised in a study of the vibration of fast trains in Germany [7, 8], associated with the company SFE GmbH in Berlin. Quadratic real and complex T-palindromic QEPs also arise in the mathematical modeling and numerical simulation of the behavior of periodic surface acoustic wave (SAW) filters [28]. The computation of the Crawford number [6], associated with the perturbation analysis of symmetric generalized eigenvalue problems, produces an $\mathrm{H}$-palindromic QEP, i.e., $B^{H}=A$ and $C^{H}=C$, where $\mathrm{H}$ stands for the conjugate transpose. The study of corner singularities in anisotropic elastic materials [1, 2, 12, 21,22] and gyroscopic systems [27] leads to T-even QEPs, i.e., $B^{T}=B, C^{T}=-C$ and $A^{T}=A$. For other QEPs with symmetry in the spectrum, see [17].

A standard approach for solving the palindromic QEP is to transform it to a $2 n \times 2 n$ linearized eigenvalue problem and compute its generalized Schur form (see [27]). However, the symplectic property of eigenvalues of (1) is not preserved generally, producing large numerical errors [11]. Recently, some pioneering work $[8,15,18]$ discovered that the T-palindromic QEP could be linearized into the form $\lambda Z^{T}+Z$, which preserves symplecticity to some extent, and suggested some structure-preserving methods to solve the linearized eigenvalue problem. Later, a $Q R$-like algorithm [23], a Jacobi-type method [8], a generalized Laub trick [16] and a $U R V$-decomposition-based structured method of cubic complexity [24] have been proposed for the palindromic linear pencil $\lambda Z^{T}+Z$. Another T-symplectic linearization $\mathcal{M}-\lambda \mathcal{L}$ of the T-palindromic QEP has been developed in [10] and two structure-preserving methods based on Patel's and Arnoldi methods are proposed to solve the T-symplectic eigenvalue problem.

*Version April 23, 2008

†School of Mathematical Sciences, Building 28, Monash University, VIC 3800, Australia; eric.chu@sci.monash.edu.au

${ }^{\ddagger}$ Department of Mathematics, National Taiwan Normal University, Taipei 11677, Taiwan; min@math.ntnu.edu.tw

$\S$ Department of Mathematics, National Tsinghua University, Hsinchu 300, Taiwan; wwlin@am.nthu.edu.tw 
In [4], another structure-preserving doubling algorithm was developed. The T-palindromic QEP can be rewritten as a factored form

$$
Q(\lambda)=\left(\lambda A^{T}-X\right) X^{-1}(\lambda X-A)
$$

with symmetries in the spectrum for some nonsingular $X$ if and only if $X$ is satisfied the following nonlinear matrix equation

$$
A^{T} X^{-1} A+X+C=0 .
$$

A structure-preserving doubling algorithm can then be applied to solve (2).

In this paper, we extend the structure-preserving doubling algorithms in $[4,14]$ to solve generalized palindromic quadratic eigenvalue problems.

This paper is organized as follows. The generalized structure-preserving doubling algorithm (g-SDA) is developed and analyzed in Sections 2 and 3. Application of the g-SDA to several generalized palindromic QEP is discussed in Section 4. The associated numerical examples will be presented in Section 5 and the paper is concluded in Section 6.

\section{2 g-SDA algorithm}

In this section, we shall develop a structure-preserving doubling algorithm to solve the generalized palindromic QEP. We first give the g-palindromic QEP and discuss some associated properties.

Definition 2.1 A function $g: \mathbb{C}^{n \times n} \rightarrow \mathbb{C}^{n \times n}$ is called $a(*, \varepsilon)$-homomorphism if $g\left(\alpha_{1} \Phi_{1}+\alpha_{2} \Phi_{2}\right)=$ $\alpha_{1}^{*} g\left(\Phi_{1}\right)+\alpha_{2}^{*} g\left(\Phi_{2}\right)$ and $g\left(\Phi_{1} \Phi_{2}\right)=\varepsilon g\left(\Phi_{2}\right) g\left(\Phi_{1}\right)$, for all $\Phi_{1}, \Phi_{2} \in \mathbb{C}^{n \times n}$ and $\alpha_{1}, \alpha_{2} \in \mathbb{C}$. Furthermore, $g$ preserves the singularity, i.e., $\operatorname{det}(\Phi)=0 \Leftrightarrow \operatorname{det}(g(\Phi))=0$. Here "*" denotes " $H$ " (Hermition/conjugate transpose) or " $T$ " (transpose) and $\varepsilon= \pm 1$.

Proposition 2.1 Let $g$ be a $(*, \varepsilon)$-homomorphism. Then it holds (i) $g(0)=0$ (ii) $g(I)=\varepsilon I$ (iii) $g\left(\Phi^{-1}\right)=$ $g(\Phi)^{-1}$.

Proof. (i) $g(\Phi)=g(\Phi+0)=g(\Phi)+g(0)$. Therefore, $g(0)=0$.

(ii) Let $\Phi$ be nonsingular. Then $g(\Phi)=g(\Phi \cdot I)=\varepsilon g(I) g(\Phi)$. From $\operatorname{det}(g(\Phi)) \neq 0$, it follows that $g(I)=\varepsilon I$.

(iii) $g(I)=g\left(\Phi^{-1} \cdot \Phi\right)=\varepsilon g(\Phi) g\left(\Phi^{-1}\right)$. From (ii), we get $g\left(\Phi^{-1}\right)=g(\Phi)^{-1}$.

Definition 2.2 The QEP in (1) is called a g-palindromic QEP if there is a $(*, \varepsilon)$-homomorphism $g$ such that $g(B)=A, g(C)=C$ and $g(A)=B$. Moreover, $C$ is called $g$-symmetric and $A$ and $B$ are said to be g-related, denoted by $A \stackrel{g}{\sim} B$.

Under the above definitions, we have the following property of symmetry in the spectrum for the above g-palindromic quadratic pencil $Q(\lambda)$.

Theorem 2.1 Let $Q(\lambda)$ be a g-palindromic quadratic pencil. We have $\lambda \in \sigma(Q(\lambda))$ if and only if $1 / \lambda^{*} \in$ $\sigma(Q(\lambda))$. Here we follow the convention that 0 and $\infty$ are reciprocal to each other.

Proof. Assume without loss of generality that $\lambda^{*} \neq 0$, otherwise the result becomes trivial. From Definitions 2.1 and 2.2 , it follows that

$$
\begin{aligned}
0 & =\operatorname{det}\left(\lambda^{2} B+\lambda C+A\right)=\operatorname{det}\left[g\left(\lambda^{2} B+\lambda C+A\right)\right] \\
& =\operatorname{det}\left[\left(\lambda^{*}\right)^{2} g(B)+\lambda^{*} g(C)+g(A)\right]=\operatorname{det}\left[A+\frac{1}{\lambda^{*}} C+\frac{1}{\left(\lambda^{*}\right)^{2}} B\right] .
\end{aligned}
$$


A (g-palindromic) quadratic pencil can be rewritten as

$$
\lambda^{2} B+\lambda C+A=(\lambda B+X) X^{-1}(\lambda X+A)=\lambda^{2} B+\lambda\left(X+B X^{-1} A\right)+A .
$$

Then we introduce a g-nonlinear matrix equation (g-NME)

$$
X+B X^{-1} A=C, \quad A \stackrel{g}{\sim} B, \quad g(C)=C .
$$

If we can find a solution $X$ for (3) structurally, then the g-palindromic QEP is factorized. We then need only to solve the eigenvalue problem for the factor $\lambda B+X$, with the reciprocal eigenvalues for $\lambda X+A$ obtained free.

For a given g-NME (3), we define

$$
\mathcal{M}=\left[\begin{array}{rr}
A & 0 \\
C & -I
\end{array}\right], \quad \mathcal{L}=\left[\begin{array}{rr}
-D & I \\
B & 0
\end{array}\right] .
$$

It is easy to see that the pencil $\mathcal{M}-\lambda \mathcal{L}$ is a linearization of g-palindromic QEP (1) with $D=0$. Based on the SDA algorithm proposed in [14], one can also develop a g-SDA algorithm for solving the g-NME.

For $\mathcal{M}-\lambda \mathcal{L}$ given in (4), we compute

$$
\mathcal{M}_{*}=\left[\begin{array}{rr}
A(C-D)^{-1} & 0 \\
-B(C-D)^{-1} & I
\end{array}\right], \quad \mathcal{L}_{*}=\left[\begin{array}{rr}
I & -A(C-D)^{-1} \\
0 & B(C-D)^{-1}
\end{array}\right]
$$

which satisfies $\mathcal{M}_{*} \mathcal{L}=\mathcal{L}_{*} \mathcal{M}$. Direct calculations give rise to

$$
\widehat{\mathcal{M}} \equiv \mathcal{M}_{*} \mathcal{M}=\left[\begin{array}{rr}
\widehat{A} & 0 \\
\widehat{C} & -I
\end{array}\right], \quad \widehat{\mathcal{L}} \equiv \mathcal{L}_{*} \mathcal{L}=\left[\begin{array}{rr}
-\widehat{D} & I \\
\widehat{B} & 0
\end{array}\right],
$$

where

$$
\begin{array}{ll}
\widehat{A}=A(C-D)^{-1} A, & \widehat{B}=B(C-D)^{-1} B, \\
\widehat{C}=C-B(C-D)^{-1} A, & \widehat{D}=D+A(C-D)^{-1} B .
\end{array}
$$

Theorem 2.2 (i) The pencil $\widehat{\mathcal{M}}-\lambda \widehat{\mathcal{L}}$ has the doubling property, i.e., if

$$
\mathcal{M}\left[\begin{array}{l}
U \\
V
\end{array}\right]=\mathcal{L}\left[\begin{array}{l}
U \\
V
\end{array}\right] S
$$

where $U, V \in \mathbb{C}^{n \times m}$ and $S \in \mathbb{C}^{m \times m}$, then

$$
\widehat{\mathcal{M}}\left[\begin{array}{l}
U \\
V
\end{array}\right]=\widehat{\mathcal{L}}\left[\begin{array}{l}
U \\
V
\end{array}\right] S^{2}
$$

(ii) The quadratic pencil $\lambda^{2} \widehat{B}+\lambda \widehat{C}+\widehat{A}$ corresponding to $\widehat{\mathcal{M}}-\lambda \widehat{\mathcal{L}}$ is still a g-palindromic quadratic pencil.

Proof. (i) From (6) and the relation $\mathcal{M}_{*} \mathcal{L}=\mathcal{L}_{*} \mathcal{M}$, we have

$$
\widehat{\mathcal{M}}\left[\begin{array}{l}
U \\
V
\end{array}\right]=\mathcal{M}_{*} \mathcal{M}\left[\begin{array}{l}
U \\
V
\end{array}\right]=\mathcal{M}_{*} \mathcal{L}\left[\begin{array}{l}
U \\
V
\end{array}\right] S=\mathcal{L}_{*} \mathcal{M}\left[\begin{array}{l}
U \\
V
\end{array}\right] S=\mathcal{L}_{*} \mathcal{L}\left[\begin{array}{l}
U \\
V
\end{array}\right] S^{2} .
$$

(ii) By Definitions 2.1 and 2.2, Proposition 2.1 and $\varepsilon^{2}=1$, we have

$$
\begin{aligned}
& g(\widehat{A})=\varepsilon^{2} g(A)(g(C)-g(D))^{-1} g(A)=B(C-D)^{-1} B=\widehat{B}, \\
& g(\widehat{B})=\varepsilon^{2} g(B)(g(C)-g(D))^{-1} g(B)=A(C-D)^{-1} A=\widehat{A}, \\
& g(\widehat{C})=g(C)-\varepsilon^{2} g(A)(g(C)-g(D))^{-1} g(B)=C-B(C-D)^{-1} A=\widehat{C}, \\
& g(\widehat{D})=g(D)+\varepsilon^{2} g(B)(g(C)-g(D))^{-1} g(A)=D+A(C-D)^{-1} B=\widehat{D} .
\end{aligned}
$$

Therefore, $\lambda^{2} \widehat{B}+\lambda \widehat{C}+\widehat{A}$ is again a g-palindromic quadratic pencil.

We restate the structure-preserving doubling algorithm (SDA-2) developed in [14] as the g-SDA algorithm for solving the g-NME. 


\section{Algorithm 2.1 (g-SDA)}

Input: $A, B, C \in \mathbb{C}^{n \times n}$ with $g(A)=B, g(B)=A$ and

$g(C)=C, \tau$ (a small tolerance $)$;

Output: a g-symmetric solution $X$ to $g$-NME.

Set $k=0, A_{k}=A, B_{k}=B, C_{k}=C$ and $D_{k}=0$;

Do until convergence:

Compute $\quad A_{k+1}=A_{k}\left(C_{k}-D_{k}\right)^{-1} A_{k}$,

$B_{k+1}=B_{k}\left(C_{k}-D_{k}\right)^{-1} B_{k}$,

$C_{k+1}=C_{k}-B_{k}\left(C_{k}-D_{k}\right)^{-1} A_{k}$,

$D_{k+1}=D_{k}+A_{k}\left(C_{k}-D_{k}\right)^{-1} B_{k}$, $k=k+1$;

If $\left\|C_{k}-C_{k-1}\right\| \leq \tau\left\|C_{k}\right\|$, Stop;

End;

Set $X \leftarrow C_{k}$.

\section{Convergence of g-SDA}

First consider the special case that $B=A^{T} \in \mathbb{R}^{n \times n}$ and $C$ is real symmetric positive definite in the g-NME $(3)$. If $(\mathcal{M}, \mathcal{L})$ in $(4)$ has no unimodular eigenvalues and the g-NME (3) has a symmetric positive solution, then the convergence of the g-SDA is quadratic [14]. If $(\mathcal{M}, \mathcal{L})$ has unimodular eigenvalues with even partial multiplicities and the g-NME (3) has a symmetric positive solution, then the convergence is globally linear with rate $1 / 2[3]$.

In this section, we shall discuss the convergence of the g-SDA for the general case of the g-NME. The quadratic convergence of the g-SDA algorithm, when no eigenvalues of the matrix pair $(\mathcal{M}, \mathcal{L})$ in $(4)$ lies on the unit circle, follows from Theorem 2.2. We shall concentrate on the more general case, assuming the following:

$(\mathcal{H})$ The partial multiplicities of $(\mathcal{M}, \mathcal{L})$ corresponding to each unimodular eigenvalue are all even with the same sizes.

Let $J_{\omega, p}$ be the $p \times p$ Jordan block with a unimodular eigenvalue $\omega=e^{\imath \theta}$ :

$$
J_{\omega, p}=\left[\begin{array}{ccccc}
\omega & 1 & & & 0 \\
& \omega & 1 & & \\
& & \ddots & \ddots & \\
& & & \ddots & 1 \\
0 & & & & \omega
\end{array}\right] .
$$

By the result in [5, pp. 557], the $2^{k}$ power of $J_{\omega, p}$ satisfies

$$
J_{\omega, p}^{2^{k}}=\left[\begin{array}{cccc}
\gamma_{1, k} & \gamma_{2, k} & \cdots & \gamma_{p, k} \\
0 & \gamma_{1, k} & \ddots & \vdots \\
\vdots & \ddots & \ddots & \gamma_{2, k} \\
0 & \cdots & 0 & \gamma_{1, k}
\end{array}\right]
$$

where

$$
\gamma_{i, k}=\frac{2^{k}\left(2^{k}-1\right) \cdots\left(2^{k}-i+2\right)}{(i-1) !} \omega^{2^{k}-i+1} \quad(i=1, \ldots, p)
$$


For each unimodular eigenvalues $\omega_{j}=e^{\imath \theta_{j}}$ of $(\mathcal{M}, \mathcal{L})$ with an even partial multiplicity $p=2 m_{j}$, we have

$$
J_{\omega_{j}, 2 m_{j}}=\left[\begin{array}{cc}
J_{\omega_{j}, m_{j}} & \Gamma_{0, m_{j}} \\
0_{m_{j}} & J_{\omega_{j}, m_{j}}
\end{array}\right], \quad \Gamma_{0, m_{j}}=e_{m_{j}} e_{1}^{T} \quad(j=1, \ldots, r) .
$$

From the Kronecker canonical form of $(\mathcal{M}, \mathcal{L})$ (see e.g. [13]), there exist nonsingular $\mathcal{Q}$ and $\mathcal{Z}$ such that

$$
\begin{aligned}
\mathcal{Q M Z} & =\left[\begin{array}{cc}
J_{s} \oplus J_{1} & 0_{\ell} \oplus \Gamma_{0} \\
0_{n} & I_{\ell} \oplus J_{1}
\end{array}\right] \equiv J_{\mathcal{M}}, \\
\mathcal{Q L Z} & =\left[\begin{array}{cc}
I_{n} & 0_{n} \\
0_{n} & J_{s}^{*} \oplus I_{m}
\end{array}\right] \equiv J_{\mathcal{L}},
\end{aligned}
$$

where $J_{s}$ is the stable Jordan block of size $\ell\left(\rho\left(J_{s}\right)<1\right), J_{1}=J_{\omega_{1}, m_{1}} \oplus \cdots \oplus J_{\omega_{r}, m_{r}}, \Gamma_{0} \equiv \Gamma_{0, m_{1}} \oplus \cdots \oplus \Gamma_{0, m_{r}}$, $\ell=n-\left(m_{1}+\cdots+m_{r}\right) \equiv n-m$ and $\oplus$ denotes the direct sum of matrices. It is easily seen from assumption $(\mathcal{H})$ that $\operatorname{span}\{\mathcal{Z}(:, 1: n)\}$ forms the unique weakly stable deflating subspace of $(\mathcal{M}, \mathcal{L})$ corresponding to $J_{s} \oplus J_{1}$.

Since $J_{\mathcal{M}}$ and $J_{\mathcal{L}}$ commute with each other, it follows from (12a) and (12b) that

$$
\mathcal{M Z} J_{\mathcal{L}}=\mathcal{Q}^{-1} J_{\mathcal{L}} J_{\mathcal{M}}=\mathcal{L} \mathcal{Z} J_{\mathcal{M}}
$$

On the other hand, we can interchange the roles of $\mathcal{M}$ and $\mathcal{L}$ in (12a) and (12b), and consider the pair $(\mathcal{L}, \mathcal{M})$ which has the same Kronecker structure of $(\mathcal{M}, \mathcal{L})$. Therefore, there are nonsingular matrices $\mathcal{P}$ and $\mathcal{Y}$ such that

$$
\mathcal{P} \mathcal{M Y}=J_{\mathcal{L}}, \quad \mathcal{P} \mathcal{L} \mathcal{Y}=J_{\mathcal{M}}
$$

where $\operatorname{span}\{\mathcal{Y}(:, 1: n)\}$ forms the unique weakly stable deflating subspace of $(\mathcal{L}, \mathcal{M})$ corresponding to $J_{s} \oplus J_{1}$. Similar arguments also produce

$$
\mathcal{L} \mathcal{Y} J_{\mathcal{L}}=\mathcal{M Y} J_{\mathcal{M}}
$$

Let $\left\{\left(\mathcal{M}_{k}, \mathcal{L}_{k}\right)\right\}_{k=0}^{\infty}$ be the sequence with

$$
\mathcal{M}_{k}=\left[\begin{array}{rr}
A_{k} & 0 \\
C_{k} & -I
\end{array}\right], \quad \mathcal{L}_{k}=\left[\begin{array}{rr}
-D_{k} & I \\
B_{k} & 0
\end{array}\right]
$$

where $A_{k}, B_{k}, C_{k}$ and $D_{k}$ are generated by Algorithm 2.1. With $\mathcal{M}_{0}=\mathcal{M}$ and $\mathcal{L}_{0}=\mathcal{L}$, it follows from (13), (16) and Theorem 2.2 that

$$
\mathcal{M}_{k} \mathcal{Z} J_{\mathcal{L}}^{2^{k}}=\mathcal{L}_{k} \mathcal{Z} J_{\mathcal{M}}^{2^{k}}
$$

Similarly, from (15), we have

$$
\mathcal{L}_{k} \mathcal{Y} J_{\mathcal{L}}^{2^{k}}=\mathcal{M}_{k} \mathcal{Y} J_{\mathcal{M}}^{2^{k}}
$$

With $\omega=\omega_{j}$ and $p=2 m_{j}$ as in (9) $(j=1, \ldots, r)$, using (12a) and (12b), (17) can be expressed as

$$
\mathcal{M}_{k} \mathcal{Z}\left[\begin{array}{cc}
I_{n} & 0_{n} \\
0_{n} & \left(J_{s}^{*}\right)^{2^{k}} \oplus I_{m}
\end{array}\right]=\mathcal{L}_{k} \mathcal{Z}\left[\begin{array}{cc}
J_{s}^{2^{k}} \oplus J_{1}^{2^{k}} & 0_{\ell} \oplus \Gamma_{k} \\
0_{n} & I_{\ell} \oplus J_{1}^{2^{k}}
\end{array}\right],
$$

where $\Gamma_{k} \equiv \Gamma_{k, m_{1}} \oplus \cdots \oplus \Gamma_{k, m_{r}}$ where

$$
\begin{aligned}
\Gamma_{k, m_{j}} & =\left[\begin{array}{ccccc}
\gamma_{m_{j}+1, k} & \gamma_{m_{j}+2, k} & \cdots & \gamma_{2 m_{j}-1, k} & \gamma_{2 m_{j}, k} \\
\gamma_{m_{j}, k} & \ddots & \ddots & & \gamma_{2 m_{j}-1, k} \\
\vdots & \ddots & \ddots & \ddots & \vdots \\
\gamma_{3, k} & & \ddots & \ddots & \gamma_{m_{j}+2, k} \\
\gamma_{2, k} & \gamma_{3, k} & \cdots & \gamma_{m_{j}, k} & \gamma_{m_{j}+1, k}
\end{array}\right] \\
& \equiv J_{\omega_{j}, 2 m_{j}}^{2^{k}}\left(1: m_{j}, m_{j}+1: 2 m_{j}\right),
\end{aligned}
$$


with $\gamma_{i, k}$ defined in $(10)$, for $i=2, \ldots, 2 m_{j}, j=1, \ldots, r$.

We now state a useful lemma in [9] for the convergence of the g-SDA.

Lemma 3.1 Let $J_{1} \equiv J_{\omega_{1}, m_{1}} \oplus \cdots \oplus J_{\omega_{r}, m_{r}}$ and $\Gamma_{k} \equiv \Gamma_{k, m_{1}} \oplus \cdots \oplus \Gamma_{k, m_{r}}$, where $\Gamma_{k, m_{j}}$ is defined in (20), for $j=1, \ldots, r$. Then $\Gamma_{k}$ is invertible and satisfies

$$
\left\|\Gamma_{k}^{-1} J_{1}^{2^{k}}\right\|=O\left(2^{-k}\right), \quad\left\|J_{1}^{2^{k}} \Gamma_{k}^{-1} J_{1}^{2^{k}}\right\|=O\left(2^{-k}\right), \quad \text { as } k \rightarrow \infty .
$$

Let $X$ be a solution for the g-NME (3). Then we have

$$
\left[\begin{array}{rr}
A & 0 \\
C & -I
\end{array}\right]\left[\begin{array}{c}
I \\
X
\end{array}\right]=\left[\begin{array}{ll}
0 & I \\
B & 0
\end{array}\right]\left[\begin{array}{c}
I \\
X
\end{array}\right] R
$$

That is, $\operatorname{span}\left\{\left[I, X^{T}\right]^{T}\right\}$ forms a deflating subspace of $(\mathcal{M}, \mathcal{L})$ corresponding to $R=X^{-1} A$.

Definition 3.1 A solution $X$ for the $g-N M E$ (3) is called to have property $(P)$, if (i) $\rho\left(X^{-1} A\right) \leq 1$, (ii) if $\rho\left(X^{-1} A\right)=1$, then the partial multiplicities of each unimodular eigenvalue of $X^{-1} A$ is half of that of the corresponding unimodular eigenvalue of the associated pair $(\mathcal{M}, \mathcal{L})$ in $(4)$.

If $X_{d}$ is a solution for the dual g-NME satisfying

$$
X_{d}+A X_{d}^{-1} B=C .
$$

Then we have

$$
\left[\begin{array}{ll}
0 & I \\
B & 0
\end{array}\right]\left[\begin{array}{c}
I \\
Y
\end{array}\right]=\left[\begin{array}{rr}
A & 0 \\
C & -I
\end{array}\right]\left[\begin{array}{c}
I \\
Y
\end{array}\right] R_{d}
$$

where $Y \equiv C-X_{d}$. That is $\operatorname{span}\left\{\left[I, Y^{T}\right]^{T}\right\}$ forms a deflating subspace of $(\mathcal{L}, \mathcal{M})$ corresponding to $R_{d} \equiv X_{d}^{-1} A$.

Theorem 3.1 Assume that the g-NME (3) and the dual g-NME (22) have the solutions $X$ and $Y$ with properties $(P)$, respectively. Suppose the sequence $\left\{A_{k}, B_{k}, C_{k}, D_{k}\right\}$ generated by Algorithm 2.1 is welldefined. Then it holds

(i) $\left\|A_{k}\right\|=O\left(\rho\left(J_{s}\right)^{2^{k}}\right)+O\left(2^{-k}\right) \rightarrow 0$, as $k \rightarrow \infty$,

(ii) $\left\|B_{k}\right\|=O\left(\rho\left(J_{s}\right)^{2^{k}}\right)+O\left(2^{-k}\right) \rightarrow 0$, as $k \rightarrow \infty$,

(iii) $\left\|C_{k}-X\right\|=O\left(\rho\left(J_{s}\right)^{2^{k+1}}\right)+O\left(2^{-k}\right) \rightarrow 0$, as $k \rightarrow \infty$,

(iv) $\left\|D_{k}-Y\right\|=O\left(\rho\left(J_{s}\right)^{2^{k+1}}\right)+O\left(2^{-k}\right) \rightarrow 0$, as $k \rightarrow \infty$.

Furthermore, $X$ and $Y$ are $g$-symmetric, i.e., $g(X)=X$ and $g(Y)=Y$.

Proof. From assumption $(\mathcal{H})$ for $(\mathcal{M}, \mathcal{L})$, it follows that the weakly stable deflating subspaces of $(\mathcal{M}, \mathcal{L})$ and $(\mathcal{L}, \mathcal{M})$ corresponding to $\left(J_{s} \oplus J_{1}\right)$, respectively, are unique. Partition $\mathcal{Z}$ in (13) and $\mathcal{Y}$ in (15) by

$$
\mathcal{Z}=\left[\begin{array}{ll}
Z_{1} & Z_{3} \\
Z_{2} & Z_{4}
\end{array}\right], \quad \mathcal{Y}=\left[\begin{array}{ll}
Y_{1} & Y_{3} \\
Y_{2} & Y_{4}
\end{array}\right]
$$

where $Z_{i}, Y_{i} \in \mathbb{C}^{n \times n}, i=1, \ldots, 4$. By assumptions for $X$ and $Y$ with properties (P), and from (13), (21), (15) and (23), we have

$$
\operatorname{span}\left\{\left[\begin{array}{c}
I \\
X
\end{array}\right]\right\}=\operatorname{span}\left\{\left[\begin{array}{c}
Z_{1} \\
Z_{2}
\end{array}\right]\right\}, \quad \operatorname{span}\left\{\left[\begin{array}{c}
I \\
Y
\end{array}\right]\right\}=\operatorname{span}\left\{\left[\begin{array}{c}
Y_{1} \\
Y_{2}
\end{array}\right]\right\} .
$$


Thus, $Z_{1}^{-1}$ and $Y_{1}^{-1}$ exist satisfying $X=Z_{2} Z_{1}^{-1}$ and $Y=Y_{2} Y_{1}^{-1}$.

Substituting $\left(\mathcal{M}_{k}, \mathcal{L}_{k}\right)$ of (16) and $\mathcal{Z}$ of (24) into (19), we obtain

$$
\begin{aligned}
A_{k} Z_{1} & =\left(-D_{k} Z_{1}+Z_{2}\right)\left(J_{s}^{2^{k}} \oplus J_{1}^{2^{k}}\right), \\
A_{k} Z_{3}\left(\left(J_{s}^{*}\right)^{2^{k}} \oplus I_{m}\right) & =\left(-D_{k} Z_{1}+Z_{2}\right)\left(0_{\ell} \oplus \Gamma_{k}\right)-\left(D_{k} Z_{3}-Z_{4}\right)\left(I_{\ell} \oplus J_{1}^{2^{k}}\right), \\
C_{k} Z_{1}-Z_{2} & =B_{k} Z_{1}\left(J_{s}^{2^{k}} \oplus J_{1}^{2^{k}}\right), \\
\left(C_{k} Z_{3}-Z_{4}\right)\left(\left(J_{s}^{*}\right)^{2^{k}} \oplus I_{m}\right) & =B_{k} Z_{1}\left(0_{\ell} \oplus \Gamma_{k}\right)+B_{k} Z_{3}\left(I_{\ell} \oplus J_{1}^{2^{k}}\right) .
\end{aligned}
$$

Similarly, substituting $\left(\mathcal{L}_{k}, \mathcal{M}_{k}\right)$ of (16) and $\mathcal{Y}$ of (24) into (18), we have

$$
\begin{aligned}
-D_{k} Y_{1}+Y_{2} & =A_{k} Y_{1}\left(J_{s}^{2^{k}} \oplus J_{1}^{2^{k}}\right), \\
\left(Y_{4}-D_{k} Y_{3}\right)\left(\left(J_{s}^{*}\right)^{2^{k}} \oplus I_{m}\right) & =A_{k} Y_{1}\left(0_{\ell} \oplus \Gamma_{k}\right)+A_{k} Y_{3}\left(I_{\ell} \oplus J_{1}^{2^{k}}\right), \\
B_{k} Y_{1} & =\left(C_{k} Y_{1}-Y_{2}\right)\left(J_{s}^{2^{k}} \oplus J_{1}^{2^{k}}\right), \\
B_{k} Y_{3}\left(\left(J_{s}^{*}\right)^{2^{k}} \oplus I_{m}\right) & =\left(C_{k} Y_{1}-Y_{2}\right)\left(0_{\ell} \oplus \Gamma_{k}\right)+\left(C_{k} Y_{3}-Y_{4}\right)\left(I_{\ell} \oplus J_{1}^{2^{k}}\right) .
\end{aligned}
$$

(i) Post-multiplying (25b) by $\left(0_{\ell} \oplus \Gamma_{k}^{-1} J_{1}^{2^{k}}\right) Z_{1}^{-1}$ and using (25a), we have

$$
\begin{aligned}
A_{k}\left[I-Z_{3}\left(0_{\ell} \oplus \Gamma_{k}^{-1} J_{1}^{2^{k}}\right) Z_{1}^{-1}\right]= & \left(-D_{k} Z_{1}+Z_{2}\right)\left(J_{s}^{2^{k}} \oplus 0_{m}\right) Z_{1}^{-1} \\
& +\left(Z_{4}-D_{k} Z_{3}\right)\left(0_{\ell} \oplus J_{1}^{2^{k}} \Gamma_{k}^{-1} J_{1}^{2^{k}}\right) Z_{1}^{-1} .
\end{aligned}
$$

Post-multiplying (26b) by $\left(0_{\ell} \oplus \Gamma_{k}^{-1} J_{1}^{2^{k}}\right) Y_{1}^{-1}$, we have

$$
\left(Y_{4}-D_{k} Y_{3}\right)\left(0_{\ell} \oplus \Gamma_{k}^{-1} J_{1}^{2^{k}}\right) Y_{1}^{-1}=A_{k} Y_{1}\left(0_{\ell} \oplus J_{1}^{2^{k}}\right) Y_{1}^{-1}+A_{k} Y_{3}\left(0_{\ell} \oplus J_{1}^{2^{k}} \Gamma_{k}^{-1} J_{1}^{2^{k}}\right) Y_{1}^{-1} .
$$

Using (26a), we have

$$
\begin{aligned}
& D_{k}\left[I-Y_{3}\left(0_{\ell} \oplus \Gamma_{k}^{-1} J_{1}^{2^{k}}\right) Y_{1}^{-1}\right]+Y_{4}\left(0_{\ell} \oplus \Gamma_{k}^{-1} J_{1}^{2^{k}}\right) Y_{1}^{-1} \\
= & Y-A_{k} Y_{1}\left(J_{s}^{2^{k}} \oplus 0_{m}\right) Y_{1}^{-1}+A_{k} Y_{3}\left(0_{\ell} \oplus J_{1}^{2^{k}} \Gamma_{k}^{-1} J_{1}^{2^{k}}\right) Y_{1}^{-1} .
\end{aligned}
$$

By Lemma 3.1, for $k$ sufficiently large, Equation (29) can be written as

$$
D_{k}=Y+O\left(2^{-k}\right)+A_{k}\left[O\left(\rho\left(J_{s}\right)^{2^{k}}\right)+O\left(2^{-k}\right)\right] .
$$

Substituting (30) into (27) and using Lemma 3.1 again, the sequence $\left\{A_{k}\right\}$ satisfies

$$
\left\|A_{k}\right\|=O\left(\rho\left(J_{s}\right)^{2^{k}}\right)+O\left(2^{-k}\right) \rightarrow 0, \quad \text { as } k \rightarrow \infty .
$$

(ii) Similar to (i), post-multiplying (26d) by $\left(0_{\ell} \oplus \Gamma_{k}^{-1} J_{1}^{2^{k}}\right) Y_{1}^{-1}$ and using (26c), we have

$$
\begin{aligned}
B_{k}\left[I_{n}-Y_{3}\left(0_{\ell} \oplus \Gamma_{k}^{-1} J_{1}^{2^{k}}\right) Y_{1}^{-1}\right]= & \left(C_{k} Y_{1}-Y_{2}\right)\left(J_{s}^{2^{k}} \oplus 0_{m}\right) Y_{1}^{-1} \\
& -\left(C_{k} Y_{3}-Y_{4}\right)\left(0_{\ell} \oplus J_{1}^{2^{k}} \Gamma_{k}^{-1} J_{1}^{2^{k}}\right) Y_{1}^{-1} .
\end{aligned}
$$

Post-multiplying (25d) by $\left(0_{\ell} \oplus \Gamma_{k}^{-1} J_{1}^{2^{k}}\right) Z_{1}^{-1}$ and using (25c), we have

$$
\begin{aligned}
& C_{k}\left[I-Z_{3}\left(0_{\ell} \oplus \Gamma_{k}^{-1} J_{1}^{2^{k}}\right) Z_{1}^{-1}\right]+Z_{4}\left(0_{\ell} \oplus \Gamma_{k}^{-1} J_{1}^{2^{k}}\right) Z_{1}^{-1} \\
= & X-B_{k} Z_{1}\left(J_{s}^{2^{k}} \oplus 0_{m}\right) Z_{1}^{-1}+B_{k} Z_{3}\left(0_{\ell} \oplus J_{1}^{2^{k}} \Gamma_{k}^{-1} J_{1}^{2^{k}}\right) Z_{1}^{-1} .
\end{aligned}
$$


By Lemma 3.1, for $k$ sufficiently large, (33) can be written as

$$
C_{k}=X+B_{k}\left[O\left(\rho\left(J_{s}\right)^{2^{k}}\right)+O\left(2^{-k}\right)\right]+O\left(2^{-k}\right) .
$$

Substituting (34) into (32), the sequence $\left\{B_{k}\right\}$ satisfies

$$
\left\|B_{k}\right\|=O\left(\rho\left(J_{s}\right)^{2^{k}}\right)+O\left(2^{-k}\right) \rightarrow 0, \text { as } k \rightarrow \infty .
$$

(iii) From (34) and (35), we obtain

$$
\left\|C_{k}-X\right\|=O\left(\rho\left(J_{s}\right)^{2^{k+1}}\right)+O\left(2^{-k}\right) \rightarrow 0, \text { as } k \rightarrow \infty .
$$

(iv) From (30) and (31) we show that

$$
\left\|D_{k}-Y\right\|=O\left(\rho\left(J_{s}\right)^{2^{k+1}}\right)+O\left(2^{-k}\right) \rightarrow 0, \text { as } k \rightarrow \infty .
$$

Furthermore, by Theorem 2.2, we have $g\left(C_{k}\right)=C_{k}$ and $g\left(D_{k}\right)=D_{k}$. This implies that $g(X)=X$ and $g(Y)=Y$.

We have shown that the convergence of the g-SDA is quadratic when no eigenvalues of the matrix pair $(\mathcal{M}, \mathcal{L})$ in $(4)$ lies on the unit circle.

Remark 3.1 Comparing the results in [3] with Theorem 3.1, we have the following comments.

(i) For the existence of the unique weakly stable deflating subspace of $(\mathcal{M}, \mathcal{L})$ in $(4)$, we have assumed that (a) the partial multiplicities of $(\mathcal{M}, \mathcal{L})$ corresponding to each unimodular eigenvalues are all even, and (b) if $\left\{\lambda_{1}, \lambda_{2}\right\}$ are unimodular eigenvalues of $(\mathcal{M}, \mathcal{L})$ with $\lambda_{1}=\lambda_{2}$, then the partial multiplicity of $\lambda_{1}$ must be equal to that of $\lambda_{2}$. In [3], only assumption (a) was required..

(ii) If the NME in [3] has a symmetric positive solution, then the matrices sequence produced by the SDA algorithm are well-defined and the convergence is globally linear with rate $1 / 2$. To guarantee the linear convergence of the g-SDA algorithm in Theorem 3.1, we need to add the assumptions that the solution of the dual g-NME exists and the iterates from the g-SDA algorithm are well-defined.

\section{Application to g-palindromic QEP}

\subsection{T- and H-(anti-)palindromic QEPs}

If the $(*, \varepsilon)$-homomorphism is defined by $g(\Phi)=\Phi^{*}$. Then the g-palindromic QEP (1) becomes

(i) T-palindromic QEP (*= "T"):

$$
\left(\lambda^{2} A^{T}+\lambda C+A\right) x=0 \text { with } C^{T}=C .
$$

(ii) H-palindromic QEP (*= "H"):

$$
\left(\lambda^{2} A^{H}+\lambda C+A\right) x=0 \text { with } C^{H}=C .
$$

If the $(*, \varepsilon)$-homomorphism is defined by $g(\Phi)=-\Phi^{*}$. Then the g-palindromic QEP becomes

(iii) T-anti-palindromic QEP (*= "T"):

$$
\left(\lambda^{2} A^{T}+\lambda C-A\right) x=0 \text { with } C=-C^{T} .
$$

(iv) H-anti-palindromic QEP (* = "H"):

$$
\left(\lambda^{2} A^{H}+\lambda C-A\right) x=0 \text { with } C=-C^{H} .
$$


For cases (i) and (ii), the g-SDA can be simplified to

$$
\begin{aligned}
A_{0} & =A, C_{0}=C=C^{*}, D_{0}=0, \\
A_{k+1} & =A_{k}\left(C_{k}-D_{k}\right)^{-1} A_{k}, \\
C_{k+1} & =C_{k}-A_{k}^{*}\left(C_{k}-D_{k}\right)^{-1} A_{k}, \\
D_{k+1} & =D_{k}+A_{k}\left(C_{k}-D_{k}\right)^{-1} A_{k}^{*} .
\end{aligned}
$$

For cases (iii) and (iv), the g-SDA can be simplified to

$$
\begin{aligned}
A_{0} & =A, C_{0}=C=-C^{*}, D_{0}=0, \\
A_{k+1} & =A_{k}\left(C_{k}-D_{k}\right)^{-1} A_{k}, \\
C_{k+1} & =C_{k}+A_{k}^{*}\left(C_{k}-D_{k}\right)^{-1} A_{k}, \\
D_{k+1} & =D_{k}-A_{k}\left(C_{k}-D_{k}\right)^{-1} A_{k}^{*} .
\end{aligned}
$$

\section{$4.2 *$ *palindromic_2 QEPs}

We now consider the $*$-palindromic_2 QEP

$$
\left(\lambda^{2} A^{*}+\lambda C-A\right) x=0 \text { with } C^{*}=C,
$$

and the $*$-anti-palindromic_2 QEP

$$
\left(\lambda^{2} A^{*}+\lambda C+A\right) x=0 \text { with } C^{*}=-C .
$$

The quadratic pencil in (42) can be factorized by

$$
\lambda A^{*}+\lambda C-A=\left(\lambda A^{*}+X\right) X^{-1}(\lambda X-A)
$$

where $X$ satisfies

$$
X-A^{*} X^{-1} A=C, C^{*}=C .
$$

The quadratic pencil in (43) can be factorized by

$$
\lambda A^{*}+\lambda C+A=\left(\lambda A^{*}+X\right) X^{-1}(\lambda X+A)
$$

where $X$ satisfies

$$
X+A^{*} X^{-1} A=C, C^{*}=-C .
$$

If we perform one step of Algorithm 2.1 on (44), then $X$ in (44) satisfies

$$
X+\widehat{A}^{*} X^{-1} \widehat{A}=\widehat{C}, \quad \widehat{C}^{*}=\widehat{C},
$$

where

$$
\widehat{A}=A C^{-1} A, \widehat{C}=C-A^{*} C^{-1} A, \widehat{D}=A C^{-1} A^{*} .
$$

The g-NME in (46) corresponds to the $*$-palindromic QEP

$$
\left(\lambda^{2} \widehat{A}^{*}+\lambda \widehat{C}+\widehat{A}\right) x=0, \quad \widehat{C}^{*}=\widehat{C} .
$$

If we perform one step of Algorithm 2.1 on (45), then $X$ in (45) satisfies

$$
X-\widehat{A}^{*} X^{-1} \widehat{A}=\widehat{C}, \quad \widehat{C}^{*}=-\widehat{C},
$$

where

$$
\widehat{A}=A C^{-1} A, \widehat{C}=C+A^{*} C^{-1} A, \widehat{D}=-A C^{-1} A^{*} .
$$

The g-NME in (49) corresponds to the $*$-anti-palindromic QEP

$$
\left(\lambda^{2} \widehat{A}^{*}+\lambda \widehat{C}-\widehat{A}\right) x=0, \quad \widehat{C}^{*}=-\widehat{C} .
$$

The g-SDA can then be applied to (48) and (51) as in Section 4.1. 


\section{3 *-even and odd QEPs}

We now consider the *-even and *-odd QEPs, respectively:

$$
Q(\lambda) x \equiv\left(\lambda^{2} M+\lambda G+K\right) x=0, M^{*}=M, K^{*}=K, G^{*}=-G,
$$

and

$$
Q(\lambda) x \equiv\left(\lambda^{2} M+\lambda G+K\right) x=0, M^{*}=-M, K^{*}=-K, G^{*}=G .
$$

It is well-known that $Q(\lambda)$ has the factorization

$$
Q(\lambda)=(\lambda M+M S+G)(\lambda I-S)
$$

if and only if $S$ is a solution of the quadratic matrix equation

$$
M S^{2}+G S+K=0
$$

If $\lambda \in \sigma(Q(\lambda))$, then $-\lambda^{*} \in \sigma(Q(\lambda))$. If $x_{i}$ and $y_{i}$ are, respectively, the right and left eigenvectors corresponding to $\lambda_{i}$ of $S$, i.e.,

$$
S x_{i}=\lambda_{i} x_{i}, \quad y_{i}^{*} S=\lambda_{i} y_{i}^{*}
$$

then $x_{i}$ and $\left(\lambda_{i} M+M S+G\right)^{-*} y_{i}$ are eigenvectors corresponding to $\lambda_{i}$ and $-\lambda_{i}^{*}$, respectively, of the QEP (52) or $(53)$.

It seems difficult to find the solution $S$ of (55) directly whose eigenvalues are on the right half-plane. Instead, the Cayley transformation $S=(I+Y)(I-Y)^{-1}$ is used. Equation (55) then becomes

$$
\varepsilon A^{*} Y^{2}+C Y+A=0
$$

where $A=M+K+G, C=2(M-K), \varepsilon=1$ for (52) and $\varepsilon=-1$ for (53). With $Y=-X^{-1} A$ in $(57)$, we have the NME:

$$
X+\varepsilon A^{*} X^{-1} A=C
$$

to which the g-SDA or Algorithm 2.1 can be applied.

\section{Numerical Results}

In this section, we test four numerical examples satisfying assumption $(\mathcal{H})$ in Section 3 to illustrate the convergence behavior of Algorithm 2.1. All numerical experiments are carried out using MATLAB 2007b with the machine precision eps $\approx 2.22 \times 10^{-16}$.

In the following, we state the processes of constructing matrices $A$ and $C$ in the g-palindromic QEP for Examples 5.5-5.8 so that they are H-palindromic, T-palindromic, H-anti-palindromic and T-anti-palindromic QEP, respectively, and each QEP satisfies assumption $(\mathcal{H})$.

Given matrices $A_{0}, G_{0} \in \mathbb{C}^{n \times n}$ such that the partial multiplicities of the purely imaginary eigenvalues (or real eigenvalues) of the Hamiltonian matrix

$$
\left[\begin{array}{cc}
A_{0} & -G_{0} \\
0 & -A_{0}^{H}
\end{array}\right]
$$

are all even, we construct a Hamiltonian matrix $\mathcal{H}$ as follows:

$$
\mathcal{H} \equiv\left[\begin{array}{ll}
I & V \\
0 & I
\end{array}\right]\left[\begin{array}{cc}
A_{0} & -G_{0} \\
0 & -A_{0}^{T}
\end{array}\right]\left[\begin{array}{cc}
I & -V \\
0 & I
\end{array}\right]=\left[\begin{array}{cc}
A_{0} & -A_{0} V-V A_{0}^{H}-G_{0} \\
0 & -A_{0}^{H}
\end{array}\right]
$$


where $V \in \mathbb{C}^{n \times n}$.

Using a Cayley transformation with an appropriate $\gamma$, the Hamiltonian matrix $\mathcal{H}$ in (59) can be transformed to a matrix pair $(\mathcal{M}, \mathcal{L}) \equiv(\mathcal{H}+\gamma I, \mathcal{H}-\gamma I)[19,20]$, and then equivalently simplifies to a matrix pair $\left(\mathcal{M}_{0}, \mathcal{L}_{0}\right)$. Here

$$
\mathcal{M}_{0}=\left[\begin{array}{cc}
\widehat{A}_{0} & 0 \\
0 & I
\end{array}\right], \quad \mathcal{L}_{0}=\left[\begin{array}{cc}
I & \widehat{G}_{0} \\
0 & \widehat{A}_{0}^{H}
\end{array}\right]
$$

with

$$
\begin{aligned}
& \widehat{A}_{0}=I+2 \gamma\left(A_{0}-\gamma I\right)^{-1}, \\
& \widehat{G}_{0}=2 \gamma\left(A_{0}-\gamma I\right)^{-1}\left(A_{0} V+V A_{0}^{H}+G_{0}\right)\left(A_{0}-\gamma I\right)^{-H} .
\end{aligned}
$$

If $\mathcal{H}$ has purely imaginary eigenvalues with even partial multiplicities, then we choose $\gamma>0$; otherwise, if $\mathcal{H}$ has real eigenvalues with even partial multiplicities, then we take $\gamma$ to be purely imaginary so that the matrix pair $\left(\mathcal{M}_{0}, \mathcal{L}_{0}\right)$ has unimodular eigenvalues with even partial multiplicities.

Choose $V$ so that $\widehat{G}_{0}$ is nonsingular. Then using following similarity transformation, the matrix pair $\left(\mathcal{M}_{0}, \mathcal{L}_{0}\right)$ in $(60)$ can be transformed to a matrix pair $(\widehat{\mathcal{M}}, \widehat{\mathcal{L}})$ :

$$
\widehat{\mathcal{M}} \equiv\left[\begin{array}{cc}
I & 0 \\
0 & -I
\end{array}\right]\left[\begin{array}{cc}
I & 0 \\
-\widehat{A}_{0}^{H} & I
\end{array}\right]\left[\begin{array}{cc}
\widehat{G}_{0}^{-1} & 0 \\
0 & I
\end{array}\right] \mathcal{M}_{0}\left[\begin{array}{cc}
I & 0 \\
-\widehat{G}_{0}^{-1} & I
\end{array}\right]=\left[\begin{array}{cc}
\widehat{G}_{0}^{-1} \widehat{A}_{0} & 0 \\
\widehat{A}_{0}^{H} \widehat{G}_{0}^{-1} \widehat{A}_{0}+\widehat{G}_{0}^{-1} & -I
\end{array}\right]
$$

and

$$
\widehat{\mathcal{L}} \equiv\left[\begin{array}{cc}
I & 0 \\
0 & -I
\end{array}\right]\left[\begin{array}{cc}
I & 0 \\
-\widehat{A}_{0}^{H} & I
\end{array}\right]\left[\begin{array}{cc}
\widehat{G}_{0}^{-1} & 0 \\
0 & I
\end{array}\right] \mathcal{L}_{0}\left[\begin{array}{cc}
I & 0 \\
-\widehat{G}_{0}^{-1} & I
\end{array}\right]=\left[\begin{array}{cc}
0 & I \\
\widehat{A}_{0}^{H} \widehat{G}_{0}^{-1} & 0
\end{array}\right]
$$

corresponding to a linearization of the g-palindromic QEP with $A=\widehat{G}_{0}^{-1} \widehat{A}_{0}, B=\widehat{A}_{0}^{H} \widehat{G}_{0}^{-1}$ and $C=$ $\widehat{A}_{0}^{H} \widehat{G}_{0}^{-1} \widehat{A}_{0}+\widehat{G}_{0}^{-1}$.

From the above construction, we have the following observations:

- Choosing $\gamma>0$ and $V^{H}=V$ such that $\widehat{G}_{0}^{H}=\widehat{G}_{0}$ is nonsingular, (62) can be simplified as

$$
\widehat{\mathcal{M}}=\left[\begin{array}{cc}
A & 0 \\
C & -I
\end{array}\right], \quad \widehat{\mathcal{L}}=\left[\begin{array}{cc}
0 & I \\
A^{H} & 0
\end{array}\right] \quad \text { with } C^{H}=C,
$$

corresponding to an H-palindromic QEP.

- Choosing $\gamma$ to be a purely imaginary value and $V^{H}=V$ such that $\widehat{G}_{0}^{H}=-\widehat{G}_{0}$ is nonsingular, (62) can be simplified as

$$
\widehat{\mathcal{M}}=\left[\begin{array}{cc}
A & 0 \\
C & -I
\end{array}\right], \quad \widehat{\mathcal{L}}=\left[\begin{array}{cc}
0 & I \\
-A^{H} & 0
\end{array}\right] \quad \text { with } C^{H}=-C,
$$

corresponding to an H-anti-palindromic QEP.

- Given $A_{0} \in \mathbb{R}^{n \times n}$ and $G_{0}^{T}=G_{0} \in \mathbb{C}^{n \times n}$, if we choose $\gamma>0$ and $V^{T}=V \in \mathbb{C}^{n \times n}$ such that $\widehat{G}_{0}$ is nonsingular, $\widehat{A}_{0} \in \mathbb{R}^{n \times n}, \widehat{G}_{0}^{T}=\widehat{G}_{0},\left(\widehat{G}_{0}^{-1} \widehat{A}_{0}\right)^{T}=\widehat{A}_{0}^{H} \widehat{G}_{0}^{-1}$ and $\left(\widehat{A}_{0}^{H} \widehat{G}_{0}^{-1} \widehat{A}_{0}+\widehat{G}_{0}^{-1}\right)^{T}=\widehat{A}_{0}^{H} \widehat{G}_{0}^{-1} \widehat{A}_{0}+$ $\widehat{G}_{0}^{-1}$. Consequently, then (62) can be simplified as

$$
\widehat{\mathcal{M}}=\left[\begin{array}{cc}
A & 0 \\
C & -I
\end{array}\right], \quad \widehat{\mathcal{L}}=\left[\begin{array}{cc}
0 & I \\
A^{T} & 0
\end{array}\right] \quad \text { with } C^{T}=C,
$$

corresponding to a T-palindromic QEP. 
- Given $A_{0} \in \mathbb{R}^{n \times n}$ and $G_{0}^{T}=-G_{0} \in \mathbb{C}^{n \times n}$, if we choose $\gamma>0$ and $V^{T}=-V \in \mathbb{C}^{n \times n}$ such that $\widehat{G}_{0}$ is nonsingular, then $\widehat{A}_{0} \in \mathbb{R}^{n \times n}, \widehat{G}_{0}^{T}=-\widehat{G}_{0},\left(\widehat{G}_{0}^{-1} \widehat{A}_{0}\right)^{T}=-\widehat{A}_{0}^{H} \widehat{G}_{0}^{-1}$ and $\left(\widehat{A}_{0}^{H} \widehat{G}_{0}^{-1} \widehat{A}_{0}+\widehat{G}_{0}^{-1}\right)^{T}=$ $-\left(\widehat{A}_{0}^{H} \widehat{G}_{0}^{-1} \widehat{A}_{0}+\widehat{G}_{0}^{-1}\right)$. Consequently, then (62) can be simplified as

$$
\widehat{\mathcal{M}}=\left[\begin{array}{cc}
A & 0 \\
C & -I
\end{array}\right], \quad \widehat{\mathcal{L}}=\left[\begin{array}{cc}
0 & I \\
-A^{T} & 0
\end{array}\right] \quad \text { with } C^{T}=-C,
$$

corresponding to a T-anti-palindromic QEP.

Based on above processes, we construct eight examples in the following. In Examples 5.1-5.4, no eigenvalues of the associated $Q(\lambda)$ lies on the unit circle. In Examples 5.5-5.8, $Q(\lambda)$ has unimodular eigenvalues and satisfies assumption $(\mathcal{H})$. Here, we use $\operatorname{trid}(a, b, c)$ to denote the tridiagonal matrix with the main-, suband super-diagonal elements being $a, b$ and $c$, respectively.

Example 5.1 (H-palindromic QEP without unimodular eigenvalues) Let

$$
A_{0}=\operatorname{diag}(63,12,39,-11,-12,-11) \quad \text { and } G_{0}=\operatorname{diag}(1,-5,-1,-1,-11,3) .
$$

Take $\gamma=16$ and $V=\operatorname{trid}(a, \bar{b}, b)$ with $a=[3,4,-4,4,1,-5]$ and $b=[-3-3 \imath,-4 \imath, 4+4 \imath, 4-4 \imath,-4-\imath]$, then $\widehat{G}_{0}$ is nonsingular and $C^{H}=C$.

Example 5.2 (T-palindromic QEP without unimodular eigenvalues) Let

$$
A_{0}=\operatorname{diag}(-37,41,24,-61,33,71) \text { and } G_{0}=\operatorname{diag}(10,8,1,-1,-4,1) .
$$

Take $\gamma=16$ and $V=\operatorname{trid}(a, b, b)$ with $a=[-3-2 \imath, 4+4 \imath,-5-4 \imath,-5,-5-\imath,-3-5 \imath]$ and $b=[1,4,-5,4,4]$, then $\widehat{G}_{0}$ is nonsingular and $C^{T}=C$.

Example 5.3 (H-anti-palindromic QEP without unimodular eigenvalues) Let

$A_{0}=\operatorname{diag}(60+22 \imath, 6+9 \imath, 74-27 \imath,-12+46 \imath, 9-99 \imath,-38+12 \imath)$ and $G_{0}=\operatorname{diag}(-5,6,-4,2,2,-6)$.

Take $\gamma=16 \imath$ and $V=\operatorname{trid}(a, \bar{b}, b)$ with $a=[-3,4,-5,-5,-5,-3]$ and $b=[-2+3 \imath, 4-4 \imath,-4+5 \imath, 5 \imath,-1+5 \imath]$, then $\widehat{G}_{0}$ is nonsingular and $C^{H}=-C$.

Example 5.4 (T-anti-palindromic QEP without unimodular eigenvalues) Let

$$
A_{0}=2 I_{4} \oplus\left[\begin{array}{ll}
2 & 2 \\
1 & 2
\end{array}\right] \text { and } G_{0}=\left[\begin{array}{ll}
0 & 0 \\
0 & 0
\end{array}\right] \oplus\left[\begin{array}{rr}
0 & \imath \\
-\imath & 0
\end{array}\right] \oplus\left[\begin{array}{rr}
0 & 1-\imath \\
-1+\imath &
\end{array}\right] .
$$

Take $\gamma=1$ and $V=\operatorname{trid}(0, b,-b)$ with $b=[-2-4 \imath, 4+3 \imath, 5-\imath,-2+3 \imath, 1+3 \imath]$, then $\widehat{G}_{0}^{T}=-\widehat{G}_{0}$ is nonsingular and $C^{T}=-C$.

Example 5.5 (H-palindromic QEP) Let $A_{0}$ and $G_{0}$ be $5 \times 5$ real matrices defined by

$$
A_{0}=\left[\begin{array}{ccc}
U & I_{2} & 0 \\
0 & U & 0 \\
0 & 0 & 2
\end{array}\right], G_{0}=\left[\begin{array}{ccc}
0 & 0 & 0 \\
0 & I_{2} & 0 \\
0 & 0 & 1
\end{array}\right],
$$

where $U=\left[\begin{array}{rr}0 & 2 \\ -2 & 0\end{array}\right]$. Then $\mathcal{H}$ in (59) has nonzero eigenvalues $\{2,-2\}$ and pure imaginary eigenvalues $\{2 \imath,-2 \imath\}$ with partial multiplicity 4 .

Take $\gamma=1$ and $V=\operatorname{trid}(a, \bar{b}, b)$ with $a=[-3,1,-4,0,2]$ and $b=[4+3 \imath, 2-\imath, 2+4 \imath,-1]$, then $\widehat{G}_{0}$ is nonsingular and $C^{H}=C$. The matrix pair $(\widehat{\mathcal{M}}, \widehat{\mathcal{L}})$ in $(62)$ has eigenvalues $\{3,1 / 3\}$ and unimodular eigenvalues $\{0.6+0.8 \imath, 0.6-0.8 \imath\}$ with partial multiplicity 4 . 
Example 5.6 (T-palindromic QEP) Let $A_{0}$ and $G_{0}$ be defined in (63). Take $\gamma=1$ and $V=\operatorname{trid}(a, b, b)$ with $a=[-4 \imath, 2+3 \imath,-4,4+3 \imath, 4+2 \imath]$ and $b=[-2,1,3,1]$, then $\widehat{G}_{0}$ is nonsingular and $C^{T}=C$. The matrix pair $(\widehat{\mathcal{M}}, \widehat{\mathcal{L}})$ in $(62)$ has eigenvalues $\{3,1 / 3\}$ and unimodular eigenvalues $\{0.6+0.8 \imath, 0.6-0.8 \imath\}$ with partial multiplicity 4 .

Example 5.7 (H-anti-palindromic QEP) Let $A_{0}$ and $G_{0}$ be $6 \times 6$ real matrices defined by

$$
A_{0}=\left[\begin{array}{cc}
U & I_{2} \\
0 & U
\end{array}\right] \oplus\left[\begin{array}{cc}
1+\imath & 0 \\
0 & 2+\imath
\end{array}\right], G_{0}=0_{2} \oplus I_{2} \oplus I_{2}
$$

where $U=\left[\begin{array}{ll}0 & 3 \\ 1 & 0\end{array}\right]$. Then $\mathcal{H}$ in (59) has nonzero eigenvalues $\{-1+\imath,-2+\imath, 2+\imath, 1+\imath\}$ and real eigenvalues $\{\sqrt{3},-\sqrt{3}\}$ with partial multiplicity 4 .

Take $\gamma=\imath$ and $V=\operatorname{trid}(a, \bar{b}, b)$ with $a=[4,3,-2,-1,-3,2]$ and $b=[3-4 \imath, 4-3 \imath, 2 \imath, \imath,-4+3 \imath]$, then $\widehat{G}_{0}$ is nonsingular and $C^{H}=-C$. The matrix pair $(\widehat{\mathcal{M}}, \widehat{\mathcal{L}})$ in $(62)$ has eigenvalues $\{1+2 \imath, 1+\imath, 0.2+0.4 \imath, 0.5+0.5 \imath\}$ and unimodular eigenvalues $\{1 / 2+\sqrt{3} / 2 \imath, 1 / 2-\sqrt{3} / 2 \imath\}$ with partial multiplicity 4.

Example 5.8 (T-anti-palindromic QEP) Let $A_{0}$ and $G_{0}$ be $6 \times 6$ matrices defined by

$$
A_{0}=\left[\begin{array}{cc}
U & I_{2} \\
0 & U
\end{array}\right] \oplus\left[\begin{array}{ll}
2 & 2 \\
1 & 2
\end{array}\right], G_{0}=\left[\begin{array}{ll}
0 & 0 \\
0 & 0
\end{array}\right] \oplus\left[\begin{array}{cc}
0 & \imath \\
-\imath & 0
\end{array}\right] \oplus\left[\begin{array}{cc}
0 & 1-\imath \\
\imath-1 & 0
\end{array}\right]
$$

where $U=\left[\begin{array}{rr}0 & 2 \\ -2 & 0\end{array}\right]$. Then $\mathcal{H}$ in (59) has pure imaginary eigenvalues $\{2 \imath,-2 \imath\}$ with partial multiplicity 4 .

Take $\gamma=1$ and $V=\operatorname{trid}(0, b,-b)$ with $b=[-2+3 \imath,-2+4 \imath, 3-4 \imath, 5+4 \imath, 1+\imath]$, then $\widehat{G}_{0}^{T}=-\widehat{G}_{0}$ is nonsingular and $C^{T}=-C$. The symplectic pair $(\widehat{\mathcal{M}}, \widehat{\mathcal{L}})$ in $(62)$ has unimodular eigenvalues $\{0.6+0.81,0.6-$ $0.8 \imath\}$ with partial multiplicity 4.

To measure the accuracy in solving g-NME (3), we use the "normalized" residual

$$
\text { NRes } \equiv \frac{\left\|\widetilde{X}+B \widetilde{X}^{-1} A-C\right\|_{2}}{\|\widetilde{X}\|_{2}+\left\|B \widetilde{X}^{-1} A\right\|_{2}+\|C\|_{2}},
$$

where $\tilde{X}$ is an approximate solution to the g-NME. When the approximate solution $\widetilde{X}$ has been computed, the approximate eigenpairs $\left(\lambda_{s}, x_{s}\right)$ and $\left(\lambda_{u}, x_{u}\right)$ with $\left|\lambda_{s}\right| \leq 1$ and $\left|\lambda_{u}\right| \geq 1$ of the g-palindromic QEP (1) can be computed from

$$
\begin{aligned}
& A x_{s}=-\lambda_{s} \widetilde{X} x_{s}, \\
& \widetilde{X} y=-\lambda_{u} B y, \quad x_{u}=\left(\lambda_{u} \widetilde{X}+A\right)^{-1} \widetilde{X} y .
\end{aligned}
$$

In our numerical tests, we report ITs (the numbers of iterations) and NRes for Algorithm 2.1 to illustrate its convergence and accuracy. All the numerical results for Examples 5.1-5.4 and 5.5-5.8 are shown in Tables 1 and 2 respectively.

We also show the accuracy of the approximate eigenpairs $\left(\lambda_{s}, x_{s}\right)$ and $\left(\lambda_{u}, x_{u}\right)$ by

$$
\operatorname{Res}=\left\|\left(\tilde{\lambda}^{2} B+\tilde{\lambda} C+A\right) \tilde{x}\right\|_{2},
$$

where $\left(\tilde{\lambda}_{\tilde{\lambda}}, \tilde{x}\right)$ is equal to $\left(\lambda_{s}, x_{s}\right)$ or $\left(\lambda_{u}, x_{u}\right)$, and the error between the exact and the approximate eigenvalues $\left(\lambda_{i}\right.$ and $\tilde{\lambda}_{i}$, respectively) of the g-palindromic QEP by

$$
\operatorname{Err}=\left|\lambda_{i}-\tilde{\lambda}_{i}\right|, \quad(i=1, \ldots, 2 n) .
$$

Define max.Res and max.Err to be the maximum of all Res and Err, respectively. The values of max.Res and max.Err for Examples 5.1-5.4 are shown in Table 1. 


\begin{tabular}{l|llll}
\hline Example & \multicolumn{1}{c}{5.1} & \multicolumn{1}{c}{5.2} & \multicolumn{1}{c}{5.3} & \multicolumn{1}{c}{5.4} \\
\hline NRes & $5.54 \times 10^{-17}$ & $5.71 \times 10^{-17}$ & $1.48 \times 10^{-16}$ & $1.19 \times 10^{-16}$ \\
ITs & 7 & 8 & 10 & 7 \\
max.Res & $1.48 \times 10^{-15}$ & $1.68 \times 10^{-15}$ & $2.44 \times 10^{-15}$ & $3.13 \times 10^{-15}$ \\
max.Err & $2.82 \times 10^{-15}$ & $3.63 \times 10^{-15}$ & $1.78 \times 10^{-15}$ & $4.10 \times 10^{-15}$ \\
\hline
\end{tabular}

Table 1: Numerical results of Examples 5.1-5.4.

\begin{tabular}{l|llll}
\hline Example & \multicolumn{1}{|c}{5.5} & \multicolumn{1}{c}{5.6} & \multicolumn{1}{c}{5.7} & \multicolumn{1}{c}{5.8} \\
\hline NRes & $3.52 \times 10^{-10}$ & $8.85 \times 10^{-11}$ & $4.40 \times 10^{-11}$ & $1.36 \times 10^{-14}$ \\
ITs & 21 & 18 & 21 & 21 \\
\hline
\end{tabular}

Table 2: Numerical results of Algorithm 2.1 for Examples 5.5-5.8.

As mentioned before, theoretically, the eigenvalues of (1) appear in pairs $\left(\lambda, \frac{1}{\lambda^{*}}\right)$. So, if we sort the eigenvalues in ascending order by modulus $\left(\left|\lambda_{1}\right| \leq \cdots \leq\left|\lambda_{n}\right| \leq 1 \leq\left|\lambda_{n+1}\right| \leq \cdots \leq\left|\lambda_{2 n}\right|\right)$, the $*$-product of the $i$ th and $(2 n+1-i)$ th sorted eigenvalues should be one. Therefore, we define the $*$-reciprocities of computed eigenvalues by

$$
* \operatorname{Rec}=\left|\tilde{\lambda}_{i} \tilde{\lambda}_{2 n+1-i}^{*}-1\right|, \quad \text { for } i=1, \ldots, n .
$$

The values of Res, $*$ Rec and Err for Examples 5.5-5.8 are shown in Tables 3 and 4 . Note that the *reciprocities of eigenvalues are preserved in Examples 5.1-5.4. Therefore, we omitted $*$ Rec in Table 1 .

Remark 5.1 (i) In Examples 5.5-5.8, $A_{k}, B_{k}, C_{k}$ and $D_{k}$ converge linearly, matching the results (i)-(iv) of Theorem 3.1. Furthermore, $C_{k}-D_{k}$ tends to a singular matrix and before the matrices $C_{k}-D_{k}$ approach ill-conditioning, the sequence $\left\{C_{k}\right\}$ has converged well to the solutions of the g-NME.

(ii) The forward errors of the eigenvalues of the g-palindromic QEP (1) in Examples 5.5-5.8 by using Algorithm 2.1 equal to $\sqrt[4]{e p s}$ approximately. This is due to the poor separation between the $d$-stable and $d$-unstable subspectra of $(\mathcal{M}, \mathcal{L})[25,26]$.

\begin{tabular}{l|lll|lll}
\hline & \multicolumn{3}{|c|}{ Example 5.5 } & \multicolumn{3}{c}{ Example 5.6 } \\
\cline { 2 - 7 } & Res & $*$ Rec & Err & Res & $*$ Rec & Err \\
\hline$\lambda_{1}$ & $1.6 \times 10^{-16}$ & $8.0 \times 10^{-16}$ & $1.1 \times 10^{-16}$ & $1.8 \times 10^{-15}$ & $1.0 \times 10^{-15}$ & $1.9 \times 10^{-15}$ \\
$\lambda_{10}$ & $5.9 \times 10^{-10}$ & & $2.0 \times 10^{-15}$ & $5.4 \times 10^{-10}$ & & $1.9 \times 10^{-14}$ \\
\hline$\lambda_{2}$ & $3.6 \times 10^{-14}$ & $1.1 \times 10^{-12}$ & $4.0 \times 10^{-5}$ & $7.3 \times 10^{-14}$ & $3.7 \times 10^{-12}$ & $7.4 \times 10^{-5}$ \\
$\lambda_{9}$ & $9.3 \times 10^{-14}$ & & $4.0 \times 10^{-5}$ & $1.2 \times 10^{-13}$ & & $7.4 \times 10^{-5}$ \\
\hline$\lambda_{3}$ & $4.4 \times 10^{-14}$ & $1.1 \times 10^{-12}$ & $4.0 \times 10^{-5}$ & $4.7 \times 10^{-14}$ & $3.7 \times 10^{-12}$ & $7.4 \times 10^{-5}$ \\
$\lambda_{8}$ & $9.7 \times 10^{-14}$ & & $4.0 \times 10^{-5}$ & $1.1 \times 10^{-13}$ & & $7.4 \times 10^{-5}$ \\
\hline$\lambda_{4}$ & $3.6 \times 10^{-14}$ & $4.8 \times 10^{-12}$ & $3.5 \times 10^{-5}$ & $5.2 \times 10^{-14}$ & $4.3 \times 10^{-12}$ & $4.9 \times 10^{-5}$ \\
$\lambda_{7}$ & $8.7 \times 10^{-14}$ & & $3.5 \times 10^{-5}$ & $9.4 \times 10^{-14}$ & & $4.9 \times 10^{-5}$ \\
\hline$\lambda_{5}$ & $4.3 \times 10^{-14}$ & $4.8 \times 10^{-12}$ & $3.5 \times 10^{-5}$ & $6.8 \times 10^{-14}$ & $4.3 \times 10^{-12}$ & $4.9 \times 10^{-5}$ \\
$\lambda_{6}$ & $9.0 \times 10^{-14}$ & & $3.5 \times 10^{-5}$ & $1.0 \times 10^{-13}$ & & $4.9 \times 10^{-5}$ \\
\hline
\end{tabular}

Table 3: Numerical results of Examples 5.5 and 5.6. 


\begin{tabular}{l|lll|lll}
\hline & \multicolumn{3}{|c|}{ Example 5.7 } & \multicolumn{3}{c}{ Example 5.8 } \\
\cline { 2 - 7 } & Res & $*$ Rec & Err & Res & *Rec & Err \\
\hline$\lambda_{1}$ & $1.3 \times 10^{-16}$ & $8.3 \times 10^{-16}$ & $4.7 \times 10^{-16}$ & $3.8 \times 10^{-16}$ & $9.3 \times 10^{-16}$ & $5.3 \times 10^{-16}$ \\
$\lambda_{12}$ & $1.9 \times 10^{-11}$ & & $2.1 \times 10^{-15}$ & $9.2 \times 10^{-14}$ & & $5.8 \times 10^{-15}$ \\
\hline$\lambda_{2}$ & $5.6 \times 10^{-17}$ & $6.7 \times 10^{-16}$ & $1.2 \times 10^{-16}$ & $2.8 \times 10^{-16}$ & $2.9 \times 10^{-15}$ & $6.9 \times 10^{-16}$ \\
$\lambda_{11}$ & $1.8 \times 10^{-11}$ & & $9.9 \times 10^{-16}$ & $4.3 \times 10^{-14}$ & & $6.6 \times 10^{-15}$ \\
\hline$\lambda_{3}$ & $2.1 \times 10^{-15}$ & $1.7 \times 10^{-12}$ & $6.0 \times 10^{-5}$ & $1.2 \times 10^{-15}$ & $2.3 \times 10^{-12}$ & $5.8 \times 10^{-5}$ \\
$\lambda_{10}$ & $1.0 \times 10^{-14}$ & & $6.0 \times 10^{-5}$ & $3.2 \times 10^{-16}$ & & $5.8 \times 10^{-5}$ \\
\hline$\lambda_{4}$ & $1.0 \times 10^{-14}$ & $1.7 \times 10^{-12}$ & $6.0 \times 10^{-5}$ & $6.0 \times 10^{-16}$ & $5.8 \times 10^{-12}$ & $5.8 \times 10^{-5}$ \\
$\lambda_{9}$ & $1.4 \times 10^{-14}$ & & $6.0 \times 10^{-5}$ & $7.1 \times 10^{-16}$ & & $5.8 \times 10^{-5}$ \\
\hline$\lambda_{5}$ & $3.6 \times 10^{-15}$ & $1.7 \times 10^{-12}$ & $4.3 \times 10^{-5}$ & $6.6 \times 10^{-16}$ & $5.8 \times 10^{-12}$ & $5.8 \times 10^{-5}$ \\
$\lambda_{8}$ & $8.8 \times 10^{-15}$ & & $4.3 \times 10^{-5}$ & $8.9 \times 10^{-16}$ & & $5.8 \times 10^{-5}$ \\
\hline$\lambda_{6}$ & $9.2 \times 10^{-15}$ & $1.7 \times 10^{-12}$ & $4.3 \times 10^{-5}$ & $1.3 \times 10^{-15}$ & $2.3 \times 10^{-12}$ & $5.8 \times 10^{-5}$ \\
$\lambda_{7}$ & $1.2 \times 10^{-14}$ & & $4.3 \times 10^{-5}$ & $4.6 \times 10^{-16}$ & & $5.8 \times 10^{-5}$ \\
\hline
\end{tabular}

Table 4: Numerical results of Examples 5.7 and 5.8.

\section{Conclusions}

In this paper, we factorize the g-palindromic quadratic pencil to a structured form so that the symmetry of the eigenvalues is preserved. The eigenpairs of the g-palindromic quadratic eigenvalue problems can then be computed if the associated g-nonlinear matrix equation (g-NME) can be solved structurally. For the g-NME, we have proposed a generalized structure-preserving doubling algorithm (g-SDA) and proved that its convergence is at least globally linear with rate 1/2, provided that Algorithm 2.1 does not break down and the g-NME and the dual-g-NME have solutions with property $(\mathrm{P})$. The results are confirmed by four numerical examples. Numerical experiments show that the g-SDA algorithm converges to the desired solutions efficiently and reliably.

\section{Acknowledgments}

This work is partially supported by the National Science Council and the National Center for Theoretical Sciences in Taiwan.

\section{References}

[1] T. Apel, V. Mehrmann, and D. Watkins. Structured eigenvalue methods for the computation of corner singularities in 3d anisotropic elastic structures. Computer methods in applied mechanics and engineering, 191:4459-4473, 2002.

[2] T. Apel, V. Mehrmann, and D. Watkins. Numerical solution of large scale structured polynomial eigenvalue problems. In Foundations of Computational Mathematics. Springer Verlag, 2003.

[3] C.-Y. Chiang, E. K.-W. Chu, C.-H. Guo, T.-M. Huang, W.-W. Lin, and S.-F. Xu. Convergence analysis of the doubling algorithm for several nonlinear matrix equations in the critical case. Technical report, NCTS Preprints in Mathematics, National Tsing Hua University, Hsinchu, Taiwan, 2008-3-004, 2008.

[4] E. K.-W. Chu, T.-M. Hwang, W.-W. Lin, and C.-T. Wu. Vibration of fast trains, palindromic eigenvalue problems and structure-preserving doubling algorithms. J. Comput. Appl. Math. (to appear). 
[5] G. H. Golub and C. F. Van Loan. Matrix Computations, 3rd ed. The Johns Hopkins University Press, 1996.

[6] N. J. Higham, F. Tisseur, and P. M. Van Dooren. Detecting a definite hermitian pair and a hyperbolic or elliptic quadratic eigenvalue problem, and associated nearness problems. Linear Alg. Appl., 351$352: 455-474,2002$.

[7] A. Hilliges. Numerische Lösung von quadratischen eigenwertproblemen mit Anwendungen in der Schiendynamik. Master's thesis, Technical University Berlin, Germany, July 2004.

[8] A Hilliges, C. Mehl, and V. Mehrmann. On the solution of palindramic eigenvalue problems. In Proceedings 4th European Congress on Computational Methods in Applied Sciences and Engineering (ECCOMAS), Jyväskylä, Finland, 2004.

[9] T.-M. Huang and W.-W. Lin. Structured doubling algorithms for weakly stabilizing Hermitian solutions of algebraic Riccati equations. To appear in Linear Alg. Appl.

[10] T.-M. Huang, W.-W. Lin, and J. Qian. Structured algorithms for palindromic quadratic eigenvalue problems arising in vibration of fast trains. Technical report, NCTS Preprints in Mathematics, National Tsing Hua University, Hsinchu, Taiwan, 2008-2-001, 2008.

[11] C.F. Ipsen. Accurate eigenvalues for fast trains. SIAM News, 37, 2004.

[12] V. A. Kozlov, V. G. Maz'ya, and J. Roßmann. Spectral properties of operator pencils generated by elliptic boundary value problems for the Lamé system. Rostocker Math. Kolloq., 51:5-24, 1997.

[13] W.-W. Lin, V. Mehrmann, and H. Xu. Canonical forms for Hamiltonian and Symplectic matrices and pencils. Linear Alg. Appl., 302-303:469-533, 1999.

[14] W.-W. Lin and S.-F. Xu. Convergence analysis of structure-preserving doubling algorithms for Riccatitye matrix equations. SIAM J. Matrix Anal. Appl., 28(1):26-39, 2006.

[15] D. S. Mackey, N. Mackey, C. Mehl, and V. Mehrmann. Structured polynomial eigenvalue problems: Good vibrations from good linearizations. To appear SIAM J. of Matrix Analysis and Appl., 2006.

[16] D. S. Mackey, N. Mackey, C. Mehl, and V. Mehrmann. Numerical methods for palindromic eigenvalue problems. Technical report, in preparation, 2007.

[17] D.S. Mackey, N. Mackey, C. Mehl, and V. Mehrmann. Structured polynomial eigenvalue problems: Good vibrations from good linearizations. SIAM J. Matrix Anal. Appl., 28:1029-1051, 2006.

[18] D.S. Mackey, N. Mackey, C. Mehl, and V. Mehrmann. Vector spaces of linearizations for matrix polynomials. SIAM J. Matrix Anal. Appl., 28:971-1004, 2006.

[19] V. Mehrmann. The Autonomous Linear Quadratic Control Problem. Springer-Verlag, 1991.

[20] V. Mehrmann. A step toward a unified treatment of continuous and discrete time control problems. Linear Alg. Appl., 241-243:749-779, 1996.

[21] V. Mehrmann and D. Watkins. Structure-preserving methods for computing eigenpairs of large sparse skew-Hamiltonian/Hamiltonian pencils. SIAM J. Sci. Comput., 22:1905-1925, 2001.

[22] H. Schmitz, K. Volk, and W. L. Wendland. On three-dimensional singularities of elastic fields near vertices. Numer. Methods Partial Differ. Equations, 9:323-337, 1993.

[23] C. Schröder. A QR-like algorithm for the palindromic eigenvalue problem. Technical report, Preprint 388, TU Berlin, Matheon, Germany, 2007. 
[24] C. Schröder. URV decomposition based structured methods for palindromic and even eigenvalue problems. Technical report, Preprint 375, TU Berlin, MATHEON, Germany, 2007.

[25] J.-G. Sun. Perturbation theory for algebraic Riccati equations. SIAM J. Matrix Anal. Appl., 19:39-65, 1998.

[26] J.-G. Sun. Condition numbers of algebraic Riccati equations in the Frobenius norm. Linear Alg. Appl., 350:237-261, 2002.

[27] F. Tisseur and K. Meerbergen. A survey of the quadratic eigenvalue problem. SIAM Rev., 43:234-286, 2001.

[28] S. Zaglmayr. Eigenvalue problems in saw-filter simulations. Diplomarbeit, Institute of Computational Mathematics, Johannes Kepler University Linz, Linz, Austria, 2002. 